\title{
Metal-binding propensity in the mitochondrial dynamin-related protein 1
}

Krishnendu Roy and Thomas J. Pucadyil

Indian Institute of Science Education and Research, Dr. Homi Bhabha Road, Pashan, Pune 411008,

Maharashtra, India

${ }^{*}$ Corresponding author: pucadyil@iiserpune.ac.in

Krishnendu Roy ORCID ID: 0000-0003-2731-2525

Thomas J. Pucadyil ORCID ID: 0000-0002-2907-9889

\begin{abstract}
Dynamin-related protein1 (Drp1) functions to divide mitochondria and peroxisomes by binding specific adaptor proteins and lipids, both of which are integral to the limiting organellar membrane. In efforts to understand how such multivalent interactions regulate Drp1 functions, in vitro reconstitution schemes rely on recruiting soluble portions of the adaptors appended with genetically encoded polyhistidine tags onto membranes containing $\mathrm{Ni}^{2+}$-bound chelator lipids. These strategies are facile and circumvent the challenge in working with membrane proteins but assume that binding is specific to proteins carrying the polyhistidine tag. Here, we find using chelator lipids and chelator beads that both native and recombinant Drp1 directly bind $\mathrm{Ni}^{2+}$ ions. Metal-binding therefore represents a potential strategy to deplete or purify Drp1 from native tissue lysates. Importantly, high concentrations of the metal in solution inhibit GTP hydrolysis and renders Drp1 inactive in membrane fission. Together, our results emphasize a metal-binding propensity, which could significantly impact Drp1 functions.
\end{abstract}

\section{Keywords}

Protein-lipid interactions, transition metal binding, Drp1, GTPase activity, membrane fission

\section{Introduction}

The dynamin-related protein 1 (Drp1) functions to affect mitochondrial and peroxisomal division. Drp1 is a soluble protein that is recruited by specific adaptors such as Mitochondrial fission factor (Mff) and the Mitochondrial dynamics protein of $49 \mathrm{kDa}$ (Mid49) and $51 \mathrm{kDa}$ (Mid51), which are single-pass integral membrane proteins present on the outer mitochondrial membrane (Kraus et al. 2021). Drp1 interacts with these adaptor proteins through conserved interfaces on the $G$ and stalk domains as well as with membrane lipids through an approximately 100-residue-long unstructured region known as the variable domain (Ramachandran 2018; Kalia and Frost 2019; Kraus et al. 2021). Soluble members of the dynamin superfamily bind membranes and self-assemble into helical 
scaffolds with enhanced GTPase activity (Chappie and Dyda 2013). GTPase-induced conformational changes in the scaffold forces membrane constriction leading to fission (Dar et al. 2015).

Previous reconstitution approaches have relied on membrane templates to understand Drp1 functions. The simplest of these rely on lipid-based recruitment of Drp1 on to the membrane and have demonstrated Drp1's capacity for constricting and severing membrane tubes (Kamerkar et al. 2018a). In attempts to involve adaptor proteins in this scheme, but circumvent experimental challenges in working with integral membrane proteins, our group and several others have used a strategy to recruit polyhistidine (6xHis) tagged constructs of the soluble domains of the adaptors onto templates containing the $\mathrm{Ni}^{2+}$-bound chelator lipid 1,2-dioleoyl-sn-glycero-3-[( $\mathrm{N}$-(5-amino-1carboxypentyl)iminodiacetic acid)succinyl] (nickel salt) (DGS-NTA(Ni $\left.{ }^{2+}\right)$ ) (Koirala et al. 2013; Clinton et al. 2016; Osellame et al. 2016; Clinton and Mears 2017; Kamerkar et al. 2018a). However, a previous study utilizing such a strategy reported a direct interaction between Drp1 and DGS$\mathrm{NTA}\left(\mathrm{Ni}^{2+}\right)$, which was surprising because the Drp1 construct used was devoid of a 6xHis tag (Koirala et al. 2013). Such interactions could potentially complicate efforts to reconstitute Drp1 functions and we therefore probed its molecular basis.

\section{Materials and Methods}

\section{Protein Expression and Purification}

Human Drp1 (Uniprot ID: O00429-4) with a C-terminal Strepll tag in pET15b (Addgene plasmid $\# 174428$ ) was expressed in BL21(DE3) and grown in autoinduction medium at $18^{\circ} \mathrm{C}$ for 36 hours. Bacterial cells were pelleted and stored at $-40^{\circ} \mathrm{C}$. The frozen bacterial pellet was thawed in $20 \mathrm{mM}$ HEPES pH 7.4, $500 \mathrm{mM} \mathrm{NaCl}$ with a protease inhibitor cocktail tablet (Roche) and lysed by sonication in an ice-water bath. Lysates were spun at $30,000 \mathrm{xg}$ for 20 mins. The supernatant was applied to a 5 $\mathrm{ml}$ streptactin column (GE Healthcare), pre-equilibrated with the same buffer and washed extensively. Buffer was exchanged to $20 \mathrm{mM}$ HEPES pH 7.4, $150 \mathrm{mM} \mathrm{NaCl}$ and bound protein was eluted with the same buffer containing $2.5 \mathrm{mM}$ desthiobiotin (Sigma-Aldrich). Proteins were spun at 100,000x g to remove aggregates before use in assays. Protein concentration was estimated from UV absorbance at $280 \mathrm{~nm}$ from the molar extinction coefficient predicted using the Expasy ProtParam tool.

\section{Liposome Preparation}

1,2-dioleoyl-sn-glycero-3-phosphocholine (DOPC), 1',3'-bis[1,2-dioleoyl-sn-glycero-3-phospho]glycerol (18:1 cardiolipin, $\mathrm{CL})$ and 1,2-dioleoyl-sn-glycero-3-[(N-(5-amino-1carboxypentyl)iminodiacetic acid)succinyl] (nickel salt) (DGS-NTA $\left(\mathrm{Ni}^{2+}\right)$ were purchased from Avanti Polar Lipids. DOPC was the bulk lipid in all experiments. The UV-activable, diazirine-containing fluorescent lipid probe (BODIPY-diazirine PE) was prepared according to published protocols (Jose and Pucadyil 2020; Jose et al. 2020). Desired lipids were aliquoted in the required amount into a glass tube and dried under high vacuum for 30 mins to a thin film. Dried lipids were then hydrated in deionized water at $50^{\circ} \mathrm{C}$ for 30 mins, vortexed vigorously and extruded through $100 \mathrm{~nm}$ pore-size polycarbonate filters (Whatman). 


\section{Proximity-based Labeling of Membrane-Associated Proteins (PLiMAP)}

Typical experiments involved mixing liposomes containing BODIPY-diazirine PE (1 mol\%) with proteins at a 100:1 molar ratio in a final volume of $30 \mu \mathrm{l}$. The reaction was incubated in dark at room temperature for 30 mins. For experiments with $6 x$ His-mCherry, Drp1 was added to liposomes after they were incubated with the $6 x$ His-mCherry for 30 mins. The reaction mix was exposed to $365 \mathrm{~nm}$ UV light (UVP crosslinker CL-1000L) at an intensity of $200 \mathrm{mJ.cm}^{-2}$ for $1 \mathrm{~min}$. The reaction mix was then boiled with sample buffer and resolved using SDS-PAGE. Gels were first imaged for BODIPY fluorescence and later fixed and imaged for Coomassie Brilliant Blue stain on an iBright 1500 (Thermo Fisher).

\section{GTPase Assays}

Drp1 $(1 \mu \mathrm{M})$ was mixed with GTP $(1 \mathrm{mM})$ and $\mathrm{MgCl}_{2}(1 \mathrm{mM})$ in $20 \mathrm{mM} \mathrm{HEPES} \mathrm{pH} \mathrm{7.4,} 150 \mathrm{mM} \mathrm{NaCl}$ in the absence or presence of liposomes and incubated at $37^{\circ} \mathrm{C}$. Buffers contained increasing concentrations of $\mathrm{NiCl}_{2}$ to test the effect of $\mathrm{Ni}^{2+}$ on Drp1's GTPase activity. Aliquots were taken out at regular intervals, quenched with EDTA and the released inorganic phosphate was assayed with a malachite-green reagent (Leonard et al. 2005).

\section{Supported Membrane Nanotubes (SMrT) and Fluorescence Imaging}

SMrT templates were prepared as described previously (Dar et al. 2017). Briefly, lipids were aliquoted at the desired ratios and brought to a final concentration of $1 \mathrm{mM}$ in chloroform. Lipid mixes contained a small amount ( $1 \mathrm{~mol} \%$ ) of the fluorescent lipid probe pTexas-Red DHPE (Thermo Fisher). $2 \mu \mathrm{l}$ of the lipid mix was spread with a glass syringe on a PEGylated glass coverslip, dried and assembled inside an FCS2 flow chamber (Bioptechs). The flow cell was then filled with buffer to hydrate the dried lipid. $\mathrm{SMrT}$ templates were formed by extruding the hydrated membrane into narrow tubes by flowing buffer at high flow rates. Fluorescence images were recorded before and after flowing Drp1 (1 $\mu \mathrm{M})$ with GTP $(1 \mathrm{mM})$ and $\mathrm{MgCl}_{2}(1 \mathrm{mM})$ in $20 \mathrm{mM}$ HEPES pH 7.4, $150 \mathrm{mM} \mathrm{NaCl}$. Buffers contained $\mathrm{NiCl}_{2}(1 \mathrm{mM})$ to test the effect of $\mathrm{Ni}^{2+}$ on Drp1's fission activity. Imaging was carried out through 100x, 1.4 NA oilimmersion objective on an Olympus IX83 inverted microscope attached with a stable LED light source (CoolLED) and an Evolve 512 EMCCD camera (Photometrics). Image acquisition was controlled by Micro-Manager and rendered using Fiji (Schindelin et al. 2012).

\section{Preparation of Brain Lysates}

Lysates from adult goat brains were prepared as described earlier (Wu et al. 2010; Kamerkar et al. 2018b). Briefly, brain tissues were cleaned of meninges and blood vessels and homogenized in a Waring blender in breaking buffer ( $25 \mathrm{mM}$ Tris $\mathrm{pH}$ 8.0, $500 \mathrm{mM} \mathrm{KCl}, 250 \mathrm{mM}$ sucrose, $2 \mathrm{mM}$ EGTA, and $1 \mathrm{mM} \mathrm{DTT}$ ) with a protease inhibitor cocktail tablet (Roche). The lysate was spun at 160,000x $\mathrm{g}$

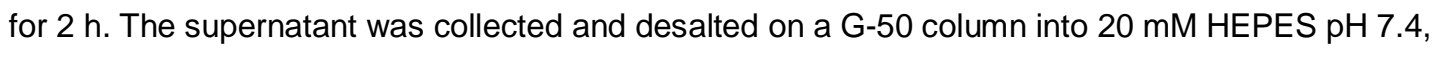
$150 \mathrm{mM} \mathrm{KCl}$. Lysates were flash-frozen with $10 \%$ glycerol in liquid nitrogen and stored at $-80^{\circ} \mathrm{C}$. Total protein content was estimated using the Pierce BCA Protein Assay Kit (Thermo Fisher). 


\section{Pulldown with Chelator Beads}

$\mathrm{Co}^{2+}$-bound NTA-sepharose beads (Takara) were stripped off the metal ion with $100 \mathrm{mM}$ EDTA and used as NTA beads or charged with $100 \mathrm{mM} \mathrm{NiCl}_{2}$ and used as $\mathrm{NTA}\left(\mathrm{Ni}^{2+}\right)$ beads. Beads were equilibrated with $20 \mathrm{mM}$ HEPES $\mathrm{pH} 7.4,150 \mathrm{mM} \mathrm{KCl}$ and incubated with $0.4 \mathrm{ml}$ of brain lysate $(0.5$ $\mathrm{mg} / \mathrm{ml}$ ) for $30 \mathrm{mins}$ at room temperature. Samples were spun at $3000 \mathrm{x}$ for $1 \mathrm{~min}$ and $20 \mu \mathrm{l}$ of the supernatant was removed. Beads were then washed 3 times with $0.4 \mathrm{ml}$ of the same buffer. The supernatant and beads were mixed with sample buffer, resolved using SDS-PAGE and probed for Drp1 using Western blotting.

\section{Western Blotting}

Proteins were transferred onto PVDF membrane and blocked with 5\% skimmed milk in TBST for $1 \mathrm{hr}$ at room temperature. Anti-Drp1 antibody (3B5, Abcam, cat. no.: Ab56788) was used at a dilution recommended by the manufacturer in blocking buffer. The membrane was incubated with the primary antibody in blocking buffer for $3 \mathrm{hrs}$ at room temperature. Subsequently, the membrane was washed with TBST and incubated with HRP-conjugated secondary antibody, diluted in blocking buffer for $1 \mathrm{hr}$ at room temperature. Blots were rinsed with TBST and developed with the chemiluminescent substrate (WestPico, Thermo Fisher Scientific) and imaged on an iBright 1500 (Thermo Fisher).

\section{Results}

The previously reported interaction between Drp1 and DGS-NTA $\left(\mathrm{Ni}^{2+}\right)$ was assayed using liposome sedimentation (Koirala et al. 2013). To confirm this interaction using sensitive methods, we turned to a recently developed crosslinking approach called Proximity-based Labeling of MembraneAssociated Proteins (PLiMAP) (Jose and Pucadyil 2020; Jose et al. 2020). PLiMAP utilizes liposomes containing a small fraction (1 mol\%) of a UV-activable, diazirine-containing fluorescent lipid probe (BODIPY-diazirine PE). Liposome binding brings proteins in proximity to the fluorescent probe and a brief exposure to UV covalently crosslinks the protein with the fluorescent probe. Liposome binding can then be quantified by in-gel fluorescence of the crosslinked protein resolved using SDS-PAGE.

We incubated recombinant $\operatorname{Drp} 1(1 \mu \mathrm{M})$ with liposomes $(100 \mu \mathrm{M})$ containing increasing concentrations of DGS-NTA $\left(\mathrm{Ni}^{2+}\right)$ and subjected them to PLiMAP. As shown in Fig. 1A (and quantitated in Fig. 1B), Drp1 is rendered fluorescent in the presence of liposomes containing DGS$\mathrm{NTA}\left(\mathrm{Ni}^{2+}\right)$, but not in its absence. Moreover, Drp1 fluorescence increases with higher concentrations of DGS-NTA $\left(\mathrm{Ni}^{2+}\right)$, implying that Drp1 binds DGS-NTA $\left(\mathrm{Ni}^{2+}\right)$. Quantitation of Drp1 fluorescence with increasing concentration of liposomes containing 10 mol\% DGS-NTA $\left(\mathrm{Ni}^{2+}\right)$ revealed an apparent binding affinity of $\sim 3 \mu \mathrm{M}$ with a hill coefficient of 1.3 (Fig. 1C, D). 

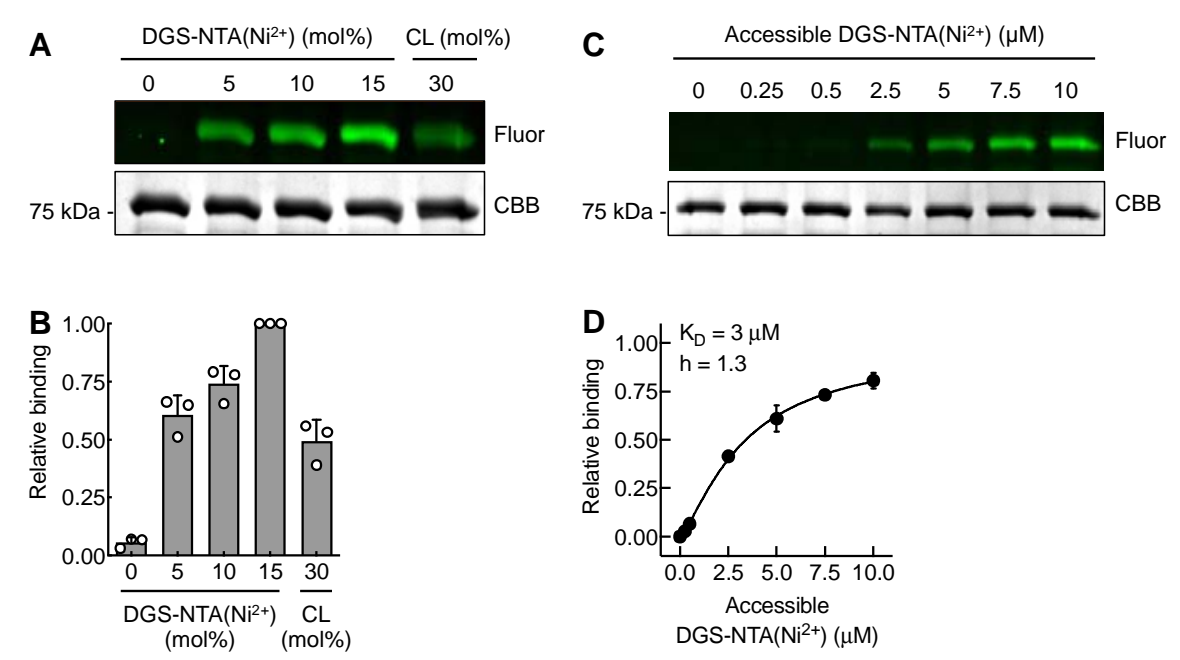

Fig. 1. Drp1 binds the chelator lipid DGS-NTA(Ni $\left.{ }^{2+}\right)$. (A) Results from a representative PLiMAP experiment showing in-gel fluorescence (Fluor) and Coomassie brilliant blue (CBB) staining of Drp1 incubated with liposomes containing the indicated concentrations of DGS-NTA $\left(\mathrm{Ni}^{2+}\right)$ and cardiolipin (CL). (B) Quantitation of in-gel fluorescence, which equates to Drp1 binding to liposomes. Data is normalized to binding seen with 15 mol\% of DGS-NTA $\left(\mathrm{Ni}^{2+}\right)$ and represents the mean \pm SD of three independent experiments. (C) Results from a representative PLiMAP experiment showing in-gel fluorescence (Fluor) and Coomassie brilliant blue (CBB) staining of Drp1 incubated with increasing concentrations of $10 \mathrm{~mol} \%$ DGS-NTA( $\left.\mathrm{Ni}^{2+}\right)$-containing liposomes. (D) Fraction of bound Drp1 plotted against the accessible DGS-NTA $\left(\mathrm{Ni}^{2+}\right)$ and fitted to a one-site specific binding with a hill slope equation. Data represents the mean $\pm S D$ of three independent experiments and is normalized to the fitted maximal binding $\left(\mathrm{B}_{\max }\right)$.

Drp1 functions have been assayed in vitro based on its ability to bind the mitochondrial lipid cardiolipin (CL) (Macdonald et al. 2014; Bustillo-Zabalbeitia et al. 2014; Stepanyants et al. 2015; Francy et al. 2017; Kamerkar et al. 2018a; Mahajan et al. 2021). Remarkably, we find that liposomes containing 5 mol\% of DGS-NTA( $\left(\mathrm{Ni}^{2+}\right)$ recruited as much Drp1 as did liposomes containing $30 \mathrm{~mol} \%$ $\mathrm{CL}$ (Fig. 1A, B), implying that Drp1 has higher affinity for DGS-NTA $\left(\mathrm{Ni}^{2+}\right)$ than $\mathrm{CL}$. Soluble dynamin proteins bind membranes and self-assemble into helical scaffolds, which in turn reorients catalytic residues in their GTP-binding G domain and stimulates their basal GTPase activity (Chappie and Dyda 2013). Thus, a stimulation in GTPase activity with membranes represents a read-out for dynamin self-assembly. Consistent with previous findings reporting that Drp1 organizes into helical scaffolds on CL-containing membranes (Francy et al. 2017), we find a 15-fold stimulation in Drp1's GTPase activity in presence of $30 \% \mathrm{CL}$-containing liposomes (Fig. 2A). Despite its strong binding to DGS-NTA $\left(\mathrm{Ni}^{2+}\right)$, Drp1's GTPase activity only showed a modest 2-fold increase with 10\% DGS-NTA $\left(\mathrm{Ni}^{2+}\right)$-containing liposomes (Fig. 2A). Drp1 functions in organelle division and we recently recreated a Drp1-catalyzed membrane fission reaction on supported membrane nanotubes (SMrT) (Kamerkar et al. 2018a). Fission requires robust stimulation of Drp1's GTPase activity. Consistent with results from GTPase assays (Fig. 2A), Drp1 caused dramatic fission of SMrT templates with 30\% CL but not with templates containing 10\% DGS-NTA(Ni $\left.{ }^{2+}\right)$ (Fig. 2B,C). These results signify that membrane 
recruitment via DGS-NTA $\left(\mathrm{Ni}^{2+}\right)$ does not mimic native Drp1-lipid interactions that allow it to function in membrane fission.
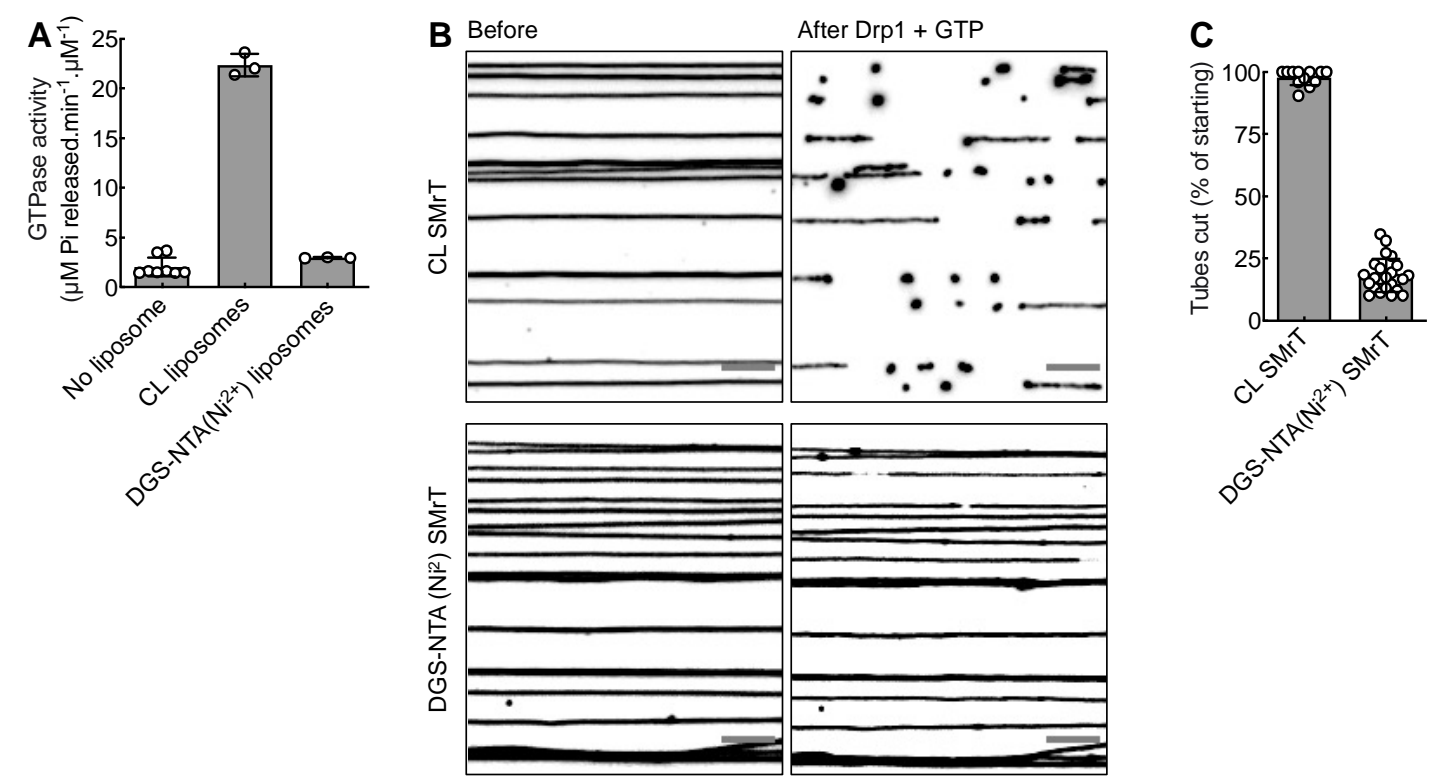

Fig. 2. DGS-NTA( $\left.\mathrm{Ni}^{2+}\right)$-recruited Drp1 is functionally inactive. (A) Drp1-catalyzed GTP hydrolysis in the absence and presence of $30 \mathrm{~mol} \% \mathrm{CL}$ - and $10 \mathrm{~mol} \%$ DGS-NTA(Ni2+)-containing liposomes. Data represent the mean \pm SD of at least three independent experiments. (B) Representative fluorescence micrographs of supported membrane nanotubes (SMrT) containing 30 mol\% CL (top) and 10 mol\% DGS-NTA(Ni2+) (bottom) imaged before and after addition of Drp1 with GTP. Fission is seen as severing of the nanotubes. Images are inverted in contrast and scale bars $=10 \mu \mathrm{m}$. (C) Quantitation of Drp1-catalyzed membrane fission of SMrTs containing 30 mol\% $\mathrm{CL}$ and $10 \mathrm{~mol} \% \mathrm{DGS}-\mathrm{NTA}\left(\mathrm{Ni}^{2+}\right)$. Data represents the mean $\pm \mathrm{SD}$ of the percentage of severed tubes in a microscope field averaged across multiple fields from two independent experiments.

Proteins that bind $\mathrm{NTA}\left(\mathrm{Ni}^{2+}\right)$ generally do so through a contiguous stretch of metal-binding residues (Bolanos-Garcia and Davies 2006; Sudan et al. 2015; Yang et al. 2019). In the case of 6xHis tags interacting with $\mathrm{NTA}\left(\mathrm{Ni}^{2+}\right)$, the NTA moiety occupies four of the six coordination sites on the metal ion while the remaining two are occupied by any two of the six imidazole moieties in the His tag (Hochuli and Piesecki 1992; Zhao et al. 2010). To understand how Drp1 binds DGS-NTA $\left(\mathrm{Ni}^{2+}\right)$, we systematically tested conditions that compete with or perturb interactions between histidine residues and NTA $\left(\mathrm{Ni}^{2+}\right)$. PLiMAP experiments where 10 mol\% DGS-NTA $\left(\mathrm{Ni}^{2+}\right)$-containing liposomes were incubated with Drp1 in presence of increasing concentrations of a random 6xHis tagged protein (6xHis-mCherry) showed a decrease in Drp1 fluorescence and an increase in 6xHis-mCherry fluorescence, implying that a $6 x$ His tag competes with Drp1 binding to DGS-NTA(Ni $\left.{ }^{2+}\right)$ (Fig. 3A,B). Interestingly, Drp1's binding to DGS-NTA $\left(\mathrm{Ni}^{2+}\right)$ could not be competed out entirely and a significant fraction remained membrane-bound even in presence of 10 -fold molar excess of the 6xHis mCherry. This has implications on the use of DGS-NTA( $\left.\mathrm{Ni}^{2+}\right)$ in reconstitution experiments with $6 x$ His-tagged constructs as very likely there will persist a Drp1 population that is directly engaged with DGS- 


\section{$\mathrm{NTA}\left(\mathrm{Ni}^{2+}\right)$. Drp1's binding to DGS-NTA $\left(\mathrm{Ni}^{2+}\right)$ was also reduced in presence of increasing} concentrations of the histidine side chain moiety Imidazole (Fig. $3 C, D$ ), indicating that this interaction is mediated by histidine residues on the protein forming a coordination complex with the $\mathrm{Ni}^{2+}$ ion (Fig. 3C,D). Lastly, striping-off the $\mathrm{Ni}^{2+}$ ion on DGS-NTA with EDTA abolished binding (Fig. 3C,D), implying that Drp1 binds the $\mathrm{Ni}^{2+}$ ion and not the chelator lipid DGS-NTA. Together, these results indicate that Drp1 is a metal-binding protein and that such interactions are mediated via histidine residues in the protein.
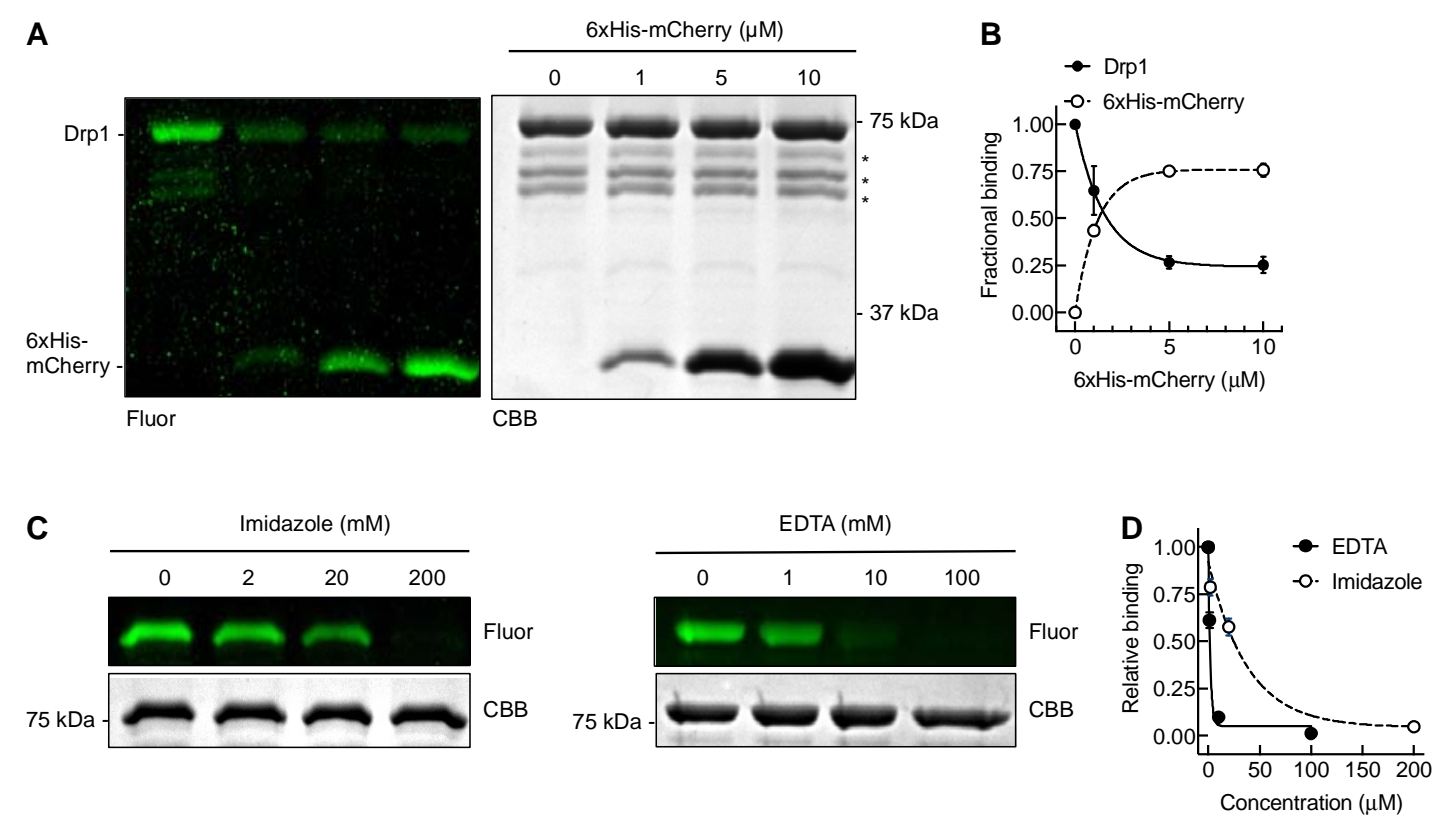

Fig. 3. Determinants of Drp1-DGS-NTA( $\left.\mathrm{Ni}^{2+}\right)$ interactions. (A) Results from a representative PLiMAP experiment showing in-gel fluorescence (Fluor) and Coomassie brilliant blue (CBB) staining of Drp1 and 6xHis mCherry with 10 mol\% DGS-NTA( $\left(\mathrm{Ni}^{2+}\right)$-containing liposomes. Asterix mark degraded Drp1. (B) Quantitation of ingel fluorescence of Drp1 and 6xHis mCherry. Data is represented as fractional liposome binding, defined as the ratio of Drp1 or 6xHis-mCherry fluorescence to the sum of Drp1 and 6xHis-mCherry fluorescence. Data represents mean \pm SD of three independent experiments. (C) Results from a representative PLiMAP experiment showing in-gel fluorescence (Fluor) and Coomassie brilliant blue (CBB) staining of Drp1 in presence of $10 \mathrm{~mol} \%$ DGS-NTA $\left(\mathrm{Ni}^{2+}\right)$ liposomes with increasing concentrations of Imidazole and EDTA. (D) Quantitation of in-gel fluorescence of Drp1 in presence of imidazole and EDTA. Data represents the mean \pm SD of three independent experiments.

Next, we tested if Drp1's propensity to bind metals chelated on a surface is apparent in a native context. For this, we incubated brain lysates with increasing amounts of $\mathrm{NTA}\left(\mathrm{Ni}^{2+}\right)$ chelator beads, sedimented them and blotted the supernatant for Drp1. As seen in Fig. 4A, brain lysates incubated with the NTA $\left(\mathrm{Ni}^{2+}\right)$ beads (but not with NTA beads alone) showed a dose-dependent reduction in Drp1 levels in the supernatant, implying efficient pull down of Drp1. Consistently, Drp1 was found bound to NTA $\left(\mathrm{Ni}^{2+}\right)$ beads but not to NTA beads (Fig. 4B). 
A

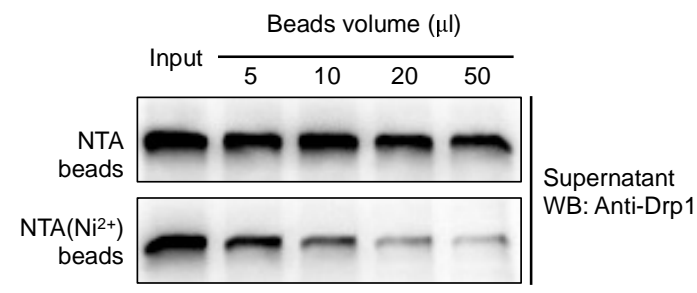

B

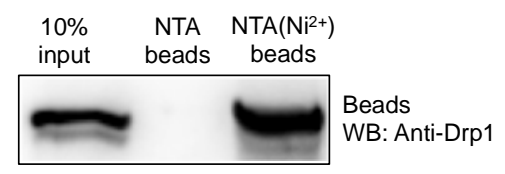

Fig. 4. Native Drp1 interacts with $\mathbf{N i}^{2+}$. (A) Representative western blots of Drp1 in the supernatant of a reaction where brain lysate was incubated with increasing amounts of NTA beads or beads charged with $\mathrm{Ni}^{2+}$. (B) Representative western blot of Drp1 pulled down with $50 \mu$ l of NTA beads or beads charged with $\mathrm{Ni}^{2+}$.

Our results show that membrane recruitment via DGS-NTA $\left(\mathrm{Ni}^{2+}\right)$ does not mimic native Drp1lipid interactions that allow it to function in membrane fission (Fig. 2). This could either be an outcome of constrained sub-optimal orientation of Drp1 on the membrane or that metal binding inhibits Drp1 functions, or both. To directly test if metal binding affects Drp1 functions, we tested GTPase and membrane fission activities on CL-containing templates in presence of free $\mathrm{Ni}^{2+}$. Remarkably, Drp1's GTPase activity with CL liposomes showed a dramatic reduction with increasing concentrations of $\mathrm{Ni}^{2+}$ added to the buffer (Fig. 5A). The stimulated GTPase activity with $\mathrm{Ni}^{2+}(1 \mathrm{mM})$ in solution reduced to basal levels seen with Drp1 in the absence of liposome. On the other hand, the basal GTPase remained largely unaffected (Fig. 5A). Consistently, presence of $\mathrm{Ni}^{2+}(1 \mathrm{mM})$ abolished Drp1's fission activity on 30\% CL-containing SMrT templates (Figs. 5B,C). Together, these results indicate that the metal significantly inhibits Drp1 functions.
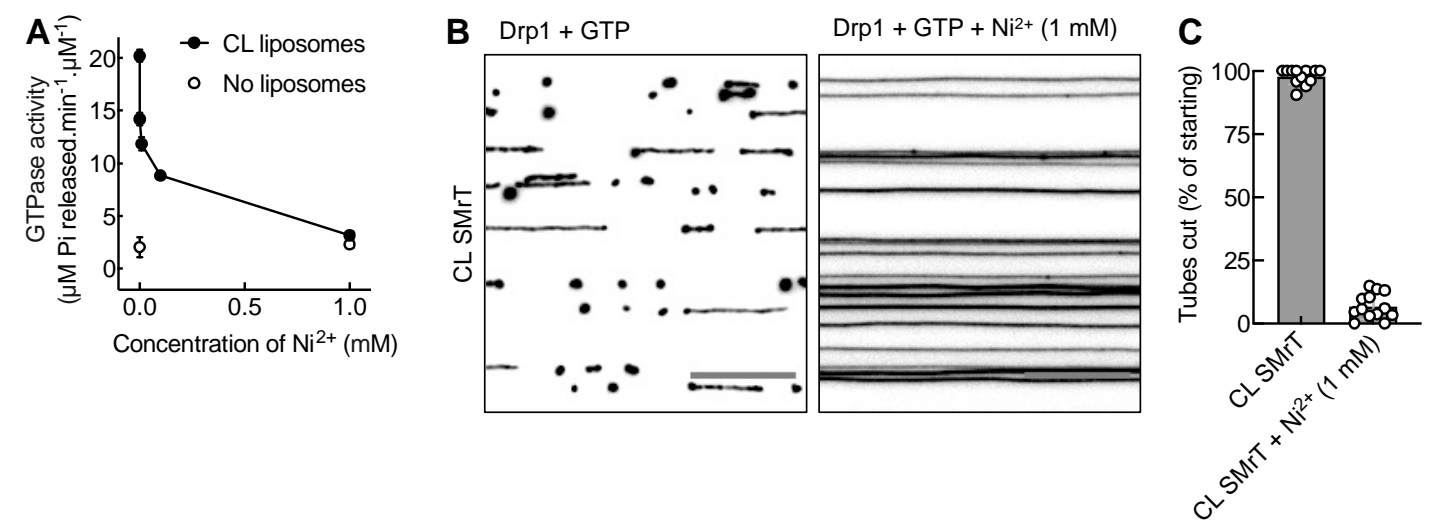

Fig. 5. $\mathbf{~ i i}^{2+}$ inactivates Drp1. (A) Drp1-catalyzed GTP hydrolysis in the absence and presence of increasing concentrations of $\mathrm{Ni}^{2+}$ assayed with and without $\mathrm{CL}$ liposomes. Data represent the mean $\pm \mathrm{SD}$ of at least two independent experiments. (B) Representative fluorescent micrographs of SMrT containing $30 \mathrm{~mol} \% \mathrm{CL}$ after addition of Drp1 $(1 \mu \mathrm{M})$ with GTP $(1 \mathrm{mM})$ in the absence (left) or presence (right) of $\mathrm{Ni}^{2+}(1 \mathrm{mM})$. Images are inverted in contrast and scale bar $=10 \mu \mathrm{m}$. (C) Quantitation of Drp1-catalyzed membrane fission in the absence and presence of $\mathrm{Ni}^{2+}(1 \mathrm{mM})$ on $30 \mathrm{~mol} \% \mathrm{CL}$ SMrTs. Data represents the mean $\pm \mathrm{SD}$ of the percentage of severed tubes in a microscope field averaged across multiple fields from two independent experiments. 


\section{Discussion}

In this report, we characterize an intrinsic metal ion-binding propensity in both native and recombinant Drp1. This property can be exploited to deplete or purify Drp1 from native tissues using standard resins used in immobilized metal affinity chromatography (IMAC). It is possible that the buffers used in lysate preparation could have removed any bound metal ion thus explaining why native Drp1 showed no binding to the IMAC resin devoid of metals. Drp1 is expressed as multiple splice variants in a tissue-specific manner (Itoh et al. 2018). Current strategies of correlating functions of these spice variants to tissue physiology rely on RT-PCR based identification of the splice variants in specific tissues. The present work utilizes the shortest splice variant (isoform 3) indicating that metal binding should be a property conserved among all splice variants. An IMAC-based pulldown and subsequent mass spectrometry could therefore represent a facile approach to identify tissuespecific expression of the splice variants and their abundance.

While we began this work with the intention of confirming an earlier reported interaction between Drp1 and metal-bound chelator lipid, we find that free metal has significant consequences on Drp1 functions. The presence of $\mathrm{Ni}^{2+}$ inactivates Drp1's stimulated GTPase activity and renders it deficient in membrane fission. Liposome-stimulated GTPase activity is attributed to self-assembly of the protein on the membrane surface and concomitant reorientation of catalytic resides in the Gdomain. Since we find that the metal selectively reduces stimulated but not basal GTPase activity, it is conceivable that the metal binding site is located on a self-assembly interface of the protein. Future work will expand the scope of the present investigation by testing binding specificity with physiologically relevant heavy metals and identification of the metal-binding site(s). Such work could have wider implications as heavy metals are known to affect mitochondrial structure and function (Dineley et al. 2003; Zischka and Borchard 2019). An exciting possibility is that such effects are mediated in part by the metal binding to and inhibiting Drp1 function.

\section{Acknowledgements}

We thank Gregor Jose for preliminary work on testing the binding of Drp1 to DGS-NTA $\left(\mathrm{Ni}^{2+}\right)$. We thank Pucadyil Lab members for valuable comments on the manuscript.

\section{Author Contributions}

KR and TJP conceptualized the study. KR performed all experiments. KR and TJP analyzed data and prepared the manuscript.

\section{Funding}

KR thanks the Council of Scientific \& Industrial Research (CSIR) for a graduate research fellowship. TJP is an International Research Scholar of the Howard Hughes Medical Institute (HHMI) and thanks the HHMI for funding support.

\section{Declarations}


bioRxiv preprint doi: https://doi.org/10.1101/2022.01.15.476484; this version posted February 10,2022 . The copyright holder for this preprint (which was not certified by peer review) is the author/funder, who has granted bioRxiv a license to display the preprint in perpetuity. It is made available under aCC-BY-NC-ND 4.0 International license.

Conflict of interest: The authors declare no conflict of interest.

\section{References}

Bolanos-Garcia VM, Davies OR (2006) Structural analysis and classification of native proteins from E. coli commonly co-purified by immobilised metal affinity chromatography. Biochimica Biophys Acta (BBA) 1760:1304-1313. https://doi.org/10.1016/j.bbagen.2006.03.027

Bustillo-Zabalbeitia I, Montessuit S, Raemy E, Basañez G, Terrones O, Martinou J-C (2014) Specific Interaction with Cardiolipin Triggers Functional Activation of Dynamin-Related Protein 1. PLoS ONE 9:e102738. https://doi.org/10.1371/journal.pone.0102738

Chappie JS, Dyda F (2013) Building a fission machine - structural insights into dynamin assembly and activation. J Cell Sci 126:2773-2784. https://doi.org/10.1242/jcs.108845

Clinton RW, Francy CA, Ramachandran R, Qi X, Mears JA (2016) Dynamin-related Protein 1 Oligomerization in Solution Impairs Functional Interactions with Membrane-anchored Mitochondrial Fission Factor*. J Biol Chem 291:478-492. https://doi.org/10.1074/jbc.m115.680025

Clinton RW, Mears JA (2017) Using Scaffold Liposomes to Reconstitute Lipid-proximal Protein-protein Interactions In Vitro. J Vis Exp. https://doi.org/10.3791/54971

Dar S, Kamerkar SC, Pucadyil TJ (2015) A high-throughput platform for real-time analysis of membrane fission reactions reveals dynamin function. Nat Cell Biol 17:1588-1596. https://doi.org/10.1038/ncb3254

Dar S, Kamerkar SC, Pucadyil TJ (2017) Use of the supported membrane tube assay system for real-time analysis of membrane fission reactions. Nat Protoc 12:390-400. https://doi.org/10.1038/nprot.2016.173

Dineley KE, Votyakova TV, Reynolds IJ (2003) Zinc inhibition of cellular energy production: implications for mitochondria and neurodegeneration. J Neurochem 85:563-570. https://doi.org/10.1046/j.14714159.2003.01678.x

Francy CA, Clinton RW, Fröhlich C, Murphy C, Mears JA (2017) Cryo-EM Studies of Drp1 Reveal Cardiolipin Interactions that Activate the Helical Oligomer. Sci Rep 7:10744. https://doi.org/10.1038/s41598-017-11008-3

Hochuli E, Piesecki S (1992) Interaction of hexahistidine fusion proteins with nitrilotriacetic acid-chelated Ni2+ ions. Methods 4:68-72. https://doi.org/10.1016/1046-2023(92)90057-f

Itoh K, Adachi Y, Yamada T, Suzuki TL, Otomo T, McBride HM, Yoshimori T, lijima M, Sesaki H (2018) A brainenriched Drp1 isoform associates with lysosomes, late endosomes, and the plasma membrane. Journal of Biological Chemistry 293:11809-11822. https://doi.org/10.1074/jbc.ra117.001253

Jose GP, Gopan S, Bhattacharyya S, Pucadyil TJ (2020) A facile, sensitive and quantitative membrane-binding assay for proteins. Traffic 21:297-305. https://doi.org/10.1111/tra.12719

Jose GP, Pucadyil TJ (2020) PLiMAP: Proximity-Based Labeling of Membrane-Associated Proteins. Curr Protoc Protein Sci $101: e 110$. https://doi.org/10.1002/cpps.110

Kalia R, Frost A (2019) Open and cut: allosteric motion and membrane fission by dynamin superfamily proteins. Mol Biol Cell 30:2097-2104. https://doi.org/10.1091/mbc.e16-10-0709

Kamerkar SC, Kraus F, Sharpe AJ, Pucadyil TJ, Ryan MT (2018a) Dynamin-related protein 1 has membrane constricting and severing abilities sufficient for mitochondrial and peroxisomal fission. Nat Commun 9:5239. https://doi.org/10.1038/s41467-018-07543-w

Kamerkar SC, Roy K, Bhattacharyya S, Pucadyil TJ (2018b) A screen for membrane fission catalysts identifies the ATPase EHD1. Biochemistry 58:65-71. https://doi.org/10.1021/acs.biochem.8b00925 
bioRxiv preprint doi: https://doi.org/10.1101/2022.01.15.476484; this version posted February 10,2022 . The copyright holder for this preprint (which was not certified by peer review) is the author/funder, who has granted bioRxiv a license to display the preprint in perpetuity. It is made available under aCC-BY-NC-ND 4.0 International license.

Koirala S, Guo Q, Kalia R, Bui HT, Eckert DM, Frost A, Shaw JM (2013) Interchangeable adaptors regulate mitochondrial dynamin assembly for membrane scission. Proc National Acad Sci 110:E1342-E1351. https://doi.org/10.1073/pnas.1300855110

Kraus F, Roy K, Pucadyil TJ, Ryan MT (2021) Function and regulation of the divisome for mitochondrial fission. Nature 590:57-66. https://doi.org/10.1038/s41586-021-03214-x

Leonard M, Song BD, Ramachandran R, Schmid SL (2005) Robust Colorimetric Assays for Dynamin's Basal and Stimulated GTPase Activities. Methods Enzymol 404:490-503. https://doi.org/10.1016/s0076-6879(05)040437

Macdonald PJ, Stepanyants N, Mehrotra N, Mears JA, Qi X, Sesaki H, Ramachandran R (2014) A dimeric equilibrium intermediate nucleates Drp1 reassembly on mitochondrial membranes for fission. Mol Biol Cell 25:1905-1915. https://doi.org/10.1091/mbc.e14-02-0728

Mahajan M, Bharambe N, Shang Y, Lu B, Mandal A, Mohan PM, Wang R, Boatz JC, Galvez JMM, Shnyrova AV, Qi X, Buck M, Wel PCA van der, Ramachandran R (2021) NMR identification of a conserved Drp1 cardiolipinbinding motif essential for stress-induced mitochondrial fission. Proc National Acad Sci 118:e2023079118. https://doi.org/10.1073/pnas.2023079118

Osellame LD, Singh AP, Stroud DA, Palmer CS, Stojanovski D, Ramachandran R, Ryan MT (2016) Cooperative and independent roles of the Drp1 adaptors Mff, MiD49 and MiD51 in mitochondrial fission. J Cell Sci 129:2170-2181

Ramachandran R (2018) Mitochondrial dynamics: The dynamin superfamily and execution by collusion. Semin Cell Dev Biol 76:201-212. https://doi.org/10.1016/j.semcdb.2017.07.039

Schindelin J, Arganda-Carreras I, Frise E, Kaynig V, Longair M, Pietzsch T, Preibisch S, Rueden C, Saalfeld S, Schmid B, Tinevez J-Y, White DJ, Hartenstein V, Eliceiri K, Tomancak P, Cardona A (2012) Fiji: an opensource platform for biological-image analysis. Nat Methods 9:676-682. https://doi.org/10.1038/nmeth.2019

Stepanyants N, Macdonald PJ, Francy CA, Mears JA, Qi X, Ramachandran R (2015) Cardiolipin's propensity for phase transition and its reorganization by dynamin-related protein 1 form a basis for mitochondrial membrane fission. Mol Biol Cell 26:3104-3116. https://doi.org/10.1091/mbc.e15-06-0330

Sudan RJJ, Kumari JLJ, Sudandiradoss C (2015) Ab Initio Coordination Chemistry for Nickel Chelation Motifs. Plos One 10:e0126787. https://doi.org/10.1371/journal.pone.0126787

Wu M, Huang B, Graham M, Raimondi A, Heuser JE, Zhuang X, Camilli PD (2010) Coupling between clathrindependent endocytic budding and F-BAR-dependent tubulation in a cell-free system. Nat Cell Biol 12:902908. https://doi.org/10.1038/ncb2094

Yang Y, Mitri K, Zhang C, Boysen RI, Hearn MTW (2019) Promiscuity of host cell proteins in the purification of histidine tagged recombinant xylanase A by IMAC procedures: A case study with a Ni2+-tacn-based IMAC system. Protein Expres Purif 162:51-61. https://doi.org/10.1016/j.pep.2019.05.009

Zhao C, Hellman LM, Zhan X, Bowman WS, Whiteheart SW, Fried MG (2010) Hexahistidine-tag-specific optical probes for analyses of proteins and their interactions. Anal Biochem 399:237-245. https://doi.org/10.1016/j.ab.2009.12.028

Zischka H, Borchard S (2019) Clinical and Translational Perspectives on WILSON DISEASE. 65-75. https://doi.org/10.1016/b978-0-12-810532-0.00008-2 